\title{
CpG oligodeoxynucleotides attenuate RORyt-mediated Th17 response by restoring histone deacetylase- 2 in cigarette smoke-exposure asthma
}

Hongtao Li ${ }^{\dagger}$, Qimei Ye ${ }^{\dagger}$, Yusen Lin, Xuena Yang, Xiaoling Zou, Hailing Yang, Wenbin Wu, Ping Meng and Tiantuo Zhang*

\begin{abstract}
Background: Cigarette smoke (CS) exposure increases corticosteroid insensitive asthma related to increased Th17 phenotype, and new treatment strategies are needed for CS-associated asthma. Histone deacetylase 2 (HDAC2), found in the airway epithelium, is critical for ameliorating glucocorticoids insensitivity. We recently demonstrated the anti-inflammatory effects of $\mathrm{CpG}$ oligodeoxynucleotides (CpG-ODNs) on CS-exposure asthma. However, the effects of CpG-ODNs on HDAC2 expression and enzymatic activity remain unclear. This study aimed to assess whether CpGODNs protect against excessive Th17 immune responses in CS-induced asthma through HDAC2-dependent mechanisms and compared their effects with those of corticosteroids.

Methods: The effects of CpG-ODNs alone and in combination with budesonide (BUD) on airway inflammation and Th2/Th17-related airway immune responses were determined using an in vivo model of CS-induced asthma and in cultured bronchial epithelial (HBE) cells administered ovalbumin (OVA) and/or cigarette smoke extract (CSE). HDAC2 and retinoid-related orphan nuclear receptor $y t$ (RORYt) expression were also assessed in mouse lung specimens and HBE cells.

Results: CpG-ODNs and BUD synergistically attenuated CS exposure asthmatic responses in vivo by modulating the influx of eosinophils and neutrophils, airway remodeling, Th2/Th17 associated cytokine and chemokine production, and airway hyperresponsiveness and blocking RORyt-mediated Th17 inflammation through induced HDAC2 expression/activity. In vitro, CpG-ODNs synergized with BUD to inhibit Th17 cytokine production in OVA- and CSE-challenged HBE cells while suppressing RORyt and increasing epithelial HDAC2 expression/activity.

Conclusions: $\mathrm{CpG}-\mathrm{ODN}$ reversed CS-induced HDAC2 downregulation and enhanced the sensitivity of CS-exposed asthmatic mice and CSE-induced HBE cells to glucocorticoid treatment. This effect may be associated with HDAC2 restoration via RORYt/IL-17 pathway regulation, suggesting that CpG-ODNs are potential corticosteroid-sparing agents for use in CS-induced asthma with Th17-biased immune conditions.
\end{abstract}

\footnotetext{
*Correspondence: zhtituli@163.com

${ }^{\dagger}$ Hongtao Li and Qimei Ye are equal contribution to the article

Department of Pulmonary and Critical Care Medicine, The Third Affiliated Hospital of Sun Yat-sen University, Institute of Respiratory Diseases of Sun

Yat-Sen University, Guangzhou, People's Republic of China
}

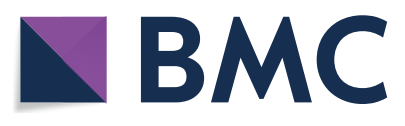

(c) The Author(s) 2021. This article is licensed under a Creative Commons Attribution 4.0 International License, which permits use, sharing, adaptation, distribution and reproduction in any medium or format, as long as you give appropriate credit to the original author(s) and the source, provide a link to the Creative Commons licence, and indicate if changes were made. The images or other third party material in this article are included in the article's Creative Commons licence, unless indicated otherwise in a credit line to the material. If material is not included in the article's Creative Commons licence and your intended use is not permitted by statutory regulation or exceeds the permitted use, you will need to obtain permission directly from the copyright holder. To view a copy of this licence, visit http://creativeco mmons.org/licenses/by/4.0/. The Creative Commons Public Domain Dedication waiver (http://creativecommons.org/publicdomain/ zero/1.0/) applies to the data made available in this article, unless otherwise stated in a credit line to the data. 
Keywords: Asthma, Histone deacetylase 2, Retinoid-related orphan nuclear receptor $\mathrm{yt}$, Th17 polarization, Corticosteroid insensitive

\section{Introduction}

Asthma is an increasingly prevalent respiratory ailment that affects at least 300 million individuals worldwide, with approximately 345,000 deaths annually [1]. Approximately $25 \%$ of the adult population in developed nations smokes, and a survey of asthma patients suggested that the rate of smoking among patients mirrored that of the general population [2]. Cigarette smoke (CS) directly and passively increases asthma susceptibility, decreases quality of life, enhances symptom severity, and exacerbates attack frequency [3]. According to several clinical studies, asthmatic smokers show poorer responses to treatment with steroids than nonsmokers [4, 5]. After exposure to CS, some cases of asthma progress to uncontrolled asthma, which is also called severe asthma.

Although inhaled corticosteroids (ICSs) are the standard therapeutic option for asthma, individuals show various responses, and most severe asthma patients may be insensitive to steroid-mediated suppression [6]. Asthma is currently considered a heterogeneous ailment, involving Th1, Th2 and Th17 cells [7]. In general, the Th2 immune response, which features eosinophil influx, substantially contributes to the development of allergic airway inflammation. Individuals with mild-to-moderate asthma mostly exhibit this disease type and can be treated with classic therapies, such as ICSs. Moreover, severe asthma is hardly manageable, even with the newest drugs. Severe asthma cases exhibit a mixed Th1/Th2 phenotype comprising a Th17 component, with elevated neutrophil rates or neutrophils plus eosinophils in the lung and sputum $[8,9]$. We previously demonstrated that CS exposure asthma was associated Th17 differentiation and budesonide (BUD) had limited effects on neutrophil infiltration in bronchoalveolar lavage fluid (BALF), which suggested CS exposure asthma may be relatively insensitive to glucocorticoids (GCs) [10]. This finding could explain why asthma patients exposed to CS directly or passively may exhibit reduced responsiveness to steroids. Approximately $5-25 \%$ of severe asthma cases show poor symptom control even after the administration of high-dose and/or systemic GCs, which contributes to nearly $50 \%$ of all asthma-associated treatment costs [11]. Accordingly, effective drugs are urgently required as mono or adjuvant therapies.

Corticosteroid insensitivity in mixed granulocytic asthma might be due to multiple factors. Classically, the molecular mechanisms of corticosteroid insensitivity mainly include the overexpression of proinflammatory transcription factors, the phosphorylation of GC receptors (GRs), and the loss of histone deacetylase-2 (HDAC2) expression [12]. A study by Li et al. suggested that HDAC2 is required for corticosteroid-associated anti-inflammation [13]. Specifically, GCs decrease inflammatory reactions via HDAC2 recruitment to the promoters of proinflammatory genes, regulating the transcription of these genes [14]. According to several studies, HDAC2 activity is decreased in alveolar macrophages, peripheral blood mononuclear cells, and bronchial biopsies of asthma patients [15-17]. Moreover, studies have also demonstrated that HDAC2 protects against airway inflammation in murine and human epithelial cells. Notably, oxidative stress induced by CS impairs HDAC2 function via ubiquitination-proteasomedependent degradation, leading to amplification of the inflammatory response and GCs insensitivity in vitro and in vivo [16, 18-21]. Therefore, GCs insensitivity correlates with $\mathrm{HDAC} 2$, suggesting that drugs that restore HDAC2 activity and expression could ameliorate GCs insensitivity.

CpG oligodeoxynucleotides (CpG-ODNs) are unmethylated $\mathrm{CpG}$ dinucleotides that mimic the immunostimulatory effects of bacterial DNA and stimulate Toll-like receptor 9 (TLR9). CpG-ODNs have shown beneficial effects in many rodent and primate models of asthma and encouraging preliminary results in clinical studies [22]. These reports suggested that CpG-ODNs induce Th1 responses and limit Th2 responses [23]. Our study and others indicated that CpG-ODNs have both potent preventive and therapeutic immunomodulatory effects on allergic inflammatory diseases [24]. The benefits of CpG-ODNs in protecting against CS-induced airway inflammation are associated with reductions in excessive CS-induced Th2/Th17 immune responses and increased Th1 responses based on our previous study [10], and further investigation of the underlying mechanism is of great interest. Retinoid-related orphan nuclear receptor $\gamma \mathrm{t}(\mathrm{ROR} \gamma \mathrm{t})$ is a transcription factor that regulates IL-17 A [25]. Despite substantial efforts to understand CpGODNs-related anti-inflammation, it remains unclear whether or how CpG-ODNs attenuate the RORytmediated Th17 response by restoring HDAC2 activity and expression, thereby ameliorating GCs insensitivity.

The current follow-up study investigated the mechanism by which CpG-ODNs regulate HDAC2 expression/activity and modulates subsequent inflammatory responses in a mouse model of CS-related asthma and 
human bronchial epithelial (HBE) cells. We established a mouse model of ovalbumin (OVA)-induced asthma after CS exposure, as well as in vitro cultures of $\mathrm{HBE}$ cells exposed to OVA and CSE, and administered CpGODNs and BUD to assess the effects of CpG-ODNs on airway inflammation and remodeling, as well as GCs insensitivity associated with HDAC2 in mice co-exposed to chronic CS and OVA. To the best of our knowledge, this was the first study to show CpG-ODNs could restore steroid sensitivity and block RORyt-induced upregulation of IL-17 in CS-induced asthma in vivo, as well as in CSE-induced HBE cells, possibly through the restoration of HDAC2 levels and activity.

\section{Materials and methods}

\section{Mice and experimental design}

Female specific pathogen free BALB/c mice (six- to seven-weeks old), provided by the Laboratory Animal Center of Southern Medical University, China (No.44,002,100,019,453), were housed under standard laboratory conditions including a $12 \mathrm{~h} / 12 \mathrm{~h}$ light-dark cycle and rodent chow and water for 3 days ad libitum.

Experimental animals were randomized to seven groups (12 animals/group), including the vehicle control, CS, OVA, OVA/CS, CpG-ODN, BUD and CpGODN/BUD groups. The latter 5 groups were sensitized and challenged with OVA. After each challenge, the mice of the latter 4 groups were exposed to CS in ventilated whole-body smoking chambers as previously described [10]. CpG-ODNs and/or BUD were administered intranasally to the last 3 groups half an hour postOVA challenge as previously described [10]. The vehicle control and CS groups were not sensitized or challenged. Moreover, the vehicle control, CS and model groups were treated with NS as negative or positive controls. The chronic CS-exposure asthmatic murine model was established as described in a previous report, with minor modifications [9]. Details are provided in the online supplementary material. A schematic diagram of the CS exposure asthmatic murine model and treatments is depicted in Additional file 1: Fig. S1.

\section{Laboratory measurements of the murine model}

Additional details of BALF sampling, the quantification of cytokines in BALF, tissue histology, immunohistochemistry, immunoblotting, serum IL-17 A and OVAspecific IgE level assessment, fluorescence microscopy, quantitative reverse transcription polymerase chain reaction (qRT-PCR), flow-cytometric analysis, and airway hyperresponsiveness (AHR) measurement are provided in the Additional file 1.

\section{HDAC2 activity}

Nuclear HDAC2 activity in the nuclear extract was measured with an HDAC2 IP \& Activity Assay Kit (BioVision Mountainview, CA, USA) according to the manufacturer's instructions. We analyzed the fluorophore and an excitation wavelength of $360 \mathrm{~nm}$ and an emission wavelength of $460 \mathrm{~nm}$ with a fluorescence plate reader.

\section{CSE preparation}

CSE preparation was performed as previous described, with minor modifications [26]. Briefly, one cigarette (each cigarette: nicotine, $1.0 \mathrm{mg}$; tar oil, $10 \mathrm{mg}$; carbon monoxide, $13 \mathrm{mg}$; Tobacco Hunan Industrial Corporation, China) was combusted, and the smoke was passed through $10 \mathrm{~mL}$ of serum-free culture medium at a rate of $5 \mathrm{~min} /$ cigarette. The $\mathrm{pH}$ of the medium was adjusted to 7.4 and diluted as indicated with culture medium. Freshly prepared CSE was used within $30 \mathrm{~min}$.

\section{Cell culture}

HBE cells, provided by the American Type Culture Collection (ATCC ${ }^{\circledR}$ PCS-300-010 ${ }^{\mathrm{TM}}$ ), underwent culture in RPMI 1640 containing 10\% fetal bovine serum (FBS). Then, the cells were administered 2.5, 5 and $10 \%$ CSE, respectively, for $6 \mathrm{~h}, 12 \mathrm{~h}, 24 \mathrm{~h}, 48$ or $72 \mathrm{~h}$ for detecting the dose/time effects of CSE on HBE cell proliferation.

\section{Cytotoxicity assay}

The cell viability in response to stimulation with of CSE for different times was examined using a standard 3-(4,5-dimethylthiazol-2-yl)-2,5-diphenyl tetrazolium bromide (MTT) assay [27]. Stimulation with CSE affected the proliferation and viability of HBE cells. A certain dose of CSE could stimulate the proliferation of HBE cells, while increasing doses of CSE induced cytotoxicity, not only inhibiting HBE cells but also inactivating them, which suggested that a specific concentration of CSE reduced the viability of HBE cells. Based on Additional file 1: Fig. S2 (see Additional file 1: Fig. S2), HBE cells exposed to $2.5 \%$ CSE for $6 \mathrm{~h}$ exhibited stable and nearly natural cell proliferation; therefore, we chose $2.5 \%$ CSE to treat HBE cells for $6 \mathrm{~h}$ to reduce experimental errors. Treatment doses of CpG-ODNs and BUD were determined in preliminary experiments.

\section{Cell treatments}

HBE cells were similarly divided into seven groups. (1) Vehicle control group: HBE cells were administered phosphate buffer saline (PBS), followed by PBS treatment. (2) CSE group: HBE cells were administered $2.5 \%$ CSE, followed by PBS treatment. (3) OVA group: 
HBE cells were administered $1 \mu \mathrm{g} / \mathrm{ml}$ OVA, followed by PBS treatment. (4) CSE/OVA group: HBE cells were administered $1 \mu \mathrm{g} / \mathrm{ml} \mathrm{OVA}$ and $2.5 \%$ CSE, followed by PBS treatment. (5) CpG-ODN group: HBE cells were administered $1 \mu \mathrm{g} / \mathrm{ml} \mathrm{OVA}$ and $2.5 \%$ CSE, followed by $5 \times 10^{-6}$ M CpG-ODN treatment. (6) BUD group: HBE cells were administered $1 \mu \mathrm{g} / \mathrm{ml}$ OVA and $2.5 \%$ CSE, followed by $10^{-8} \mathrm{M}$ BUD treatment. (7) CpG-ODN/ BUD group: HBE cells were administered $1 \mu \mathrm{g} / \mathrm{ml}$ OVA and $2.5 \%$ CSE, followed by $10^{-8} \mathrm{M}$ BUD and $5 \times 10^{-6}$ M CpG-ODN treatment.

\section{Laboratory measurements of HBE cells}

The amounts of IL-5, IL-13 (Th2 cytokines) and IL-17 A (Th17 cytokine) were assessed by specific enzyme-linked immunosorbent assay (ELISA) kits (Bioss Inc., China) as directed by the manufacturer. The relative mRNA levels of cytokines in HBE cells were assessed by qRT-PCR. The protein expression levels of IL-17 A (Invitrogen, USA), HDAC2 (Invitrogen, USA) and RORyt (Invitrogen, USA) were detected by Western blotting and immunofluorescence analysis. Flow cytometry antibodies detecting HDAC2, RORyt and IL-17 A were provided by Abcam (US). Flow cytometry was carried out on a BD Calibur instrument (BD, USA).

\section{Statistical analysis}

The data are presented as the mean \pm standard deviation (SD), and were assessed by one-way analysis of variance (ANOVA) for multiple groups, with post-hoc Tukey's multiple comparison test. GraphPad Prism 6.0 (GraphPad Software, USA) was employed for data analysis, and $p<0.05$ indicated statistical significance.

\section{Results}

Suppression of combined granulocyte inflammation, airway structural remodeling, and AHR by CpG-ODNs plus BUD in chronic CS-exposed asthmatic mice

Histological data showed that lung specimens from OVA/CS mice had substantial peribronchial and perivascular connective tissues (Additional file 1: Fig. S3a), multiple airway goblet cells containing mucus (Additional file 1: Fig. S3b, d), and peribronchial collagen deposition (Additional file 1: Fig. S3c, e). A combined granulocyte (neutrophil and eosinophil) inflammatory phenotype was confirmed as indicated by elevated Gr-1 (neutrophilspecific marker; Fig. 1b, e) and ECP (eosinophil-specific marker, Fig. 1a, d) immunohistochemical signals in the lungs, as well as marked expression of eotaxin 1 in BALF (Fig. 1c), which facilitates the recruitment of eosinophils and neutrophils [28]. The predominance of airway inflammation associated with a mixture of neutrophils and eosinophils was reduced; airway remodeling factors such as goblet cell hyperplasia and collagen accumulation were also diminished in the airways of CpG-ODNs or BUD treated mice (Additional file 1: Fig. S3) compared with those in CS-exposure asthmatic mice. Meanwhile, combined treatment with CpG-ODNs and BUD caused almost no alterations in mucus hypersecretion, negligible cell infiltration or alternations in airway wall thickness alteration, with suppression of AHR upon methacholine administration in animals with CS-exposure asthma (Fig. 1, Additional file 1: Fig. S3).

\section{Alterations in Th2/Th17 polarization and reductions in proinflammatory cytokines by CpG-ODNs and BUD in CS-associated asthmatic mice}

Th2 markers (IL-5 and IL-13) were induced, while the Th1 marker IFN- $\gamma$ was reduced after OVA $+\mathrm{CS}$ coexposure in the mouse model $(p<0.01$; Additional file 1: Fig. S4c-e and $p<0.01$; Additional file 1: Fig. S4b). Proinflammatory cytokines (IL-8 and TNF- $\alpha$ ), TGF- $\beta 1$, and serum anti-OVA IgE were also increased (all $p<0.01$, Additional file 1: Fig. S4a, f, g, h, i). These values changed substantially after treatment with CpG-ODNs (Additional file 1: Fig. S4). BUD also somewhat attenuated the CS associated increase in proinflammatory cytokines and serum anti-OVA IgE. However, we also noted that CpG-ODNs combined with BUD had additive beneficial effects on the modulation of Th1/Th2 homeostasis, proinflammatory cytokines, TGF- $\beta 1$, and anti-OVA IgE in the coadministration group (Additional file 1: Fig. 4), which showed that CpG-ODNs potentiated the effects of corticosteroids.

Th17 cells exert their effects by producing multiple inflammatory cytokines such as IL-17 A, which is known to enhance the chemotaxis of neutrophils toward bronchial epithelial cells and airway smooth muscle cells [29]. Increasing evidence has claimed that Th17-associated neutrophilic airway inflammation in the mouse is GCs insensitive [30]. As expected, Th17 cells in CS-exposure asthmatic mice were markedly elevated compared with those in the vehicle control group, according to the flow cytometry data (Fig. 2a, b). Moreover, significantly elevated protein and mRNA levels of IL-17 A in serum, lung, and BALF were found in the CS, OVA, and OVA/CS groups compared with the vehicle control group (Fig. 2cg). Both CpG-ODNs and BUD decreased the percentage of Th17-positive cells, and IL-17 mRNA and protein levels compared with those in untreated CS-induced asthmatic mice (Fig. 2a-g). Meanwhile, combined treatment with CpG-ODNs and BUD markedly reduced Th17 cells, IL-17 mRNA and protein levels compared with those in the monotherapy groups (Fig. $2 \mathrm{a}-\mathrm{g}$ ).

Taken together, these data indicated that CS-exposure associated asthma induced a Th17/Th2-type response, 


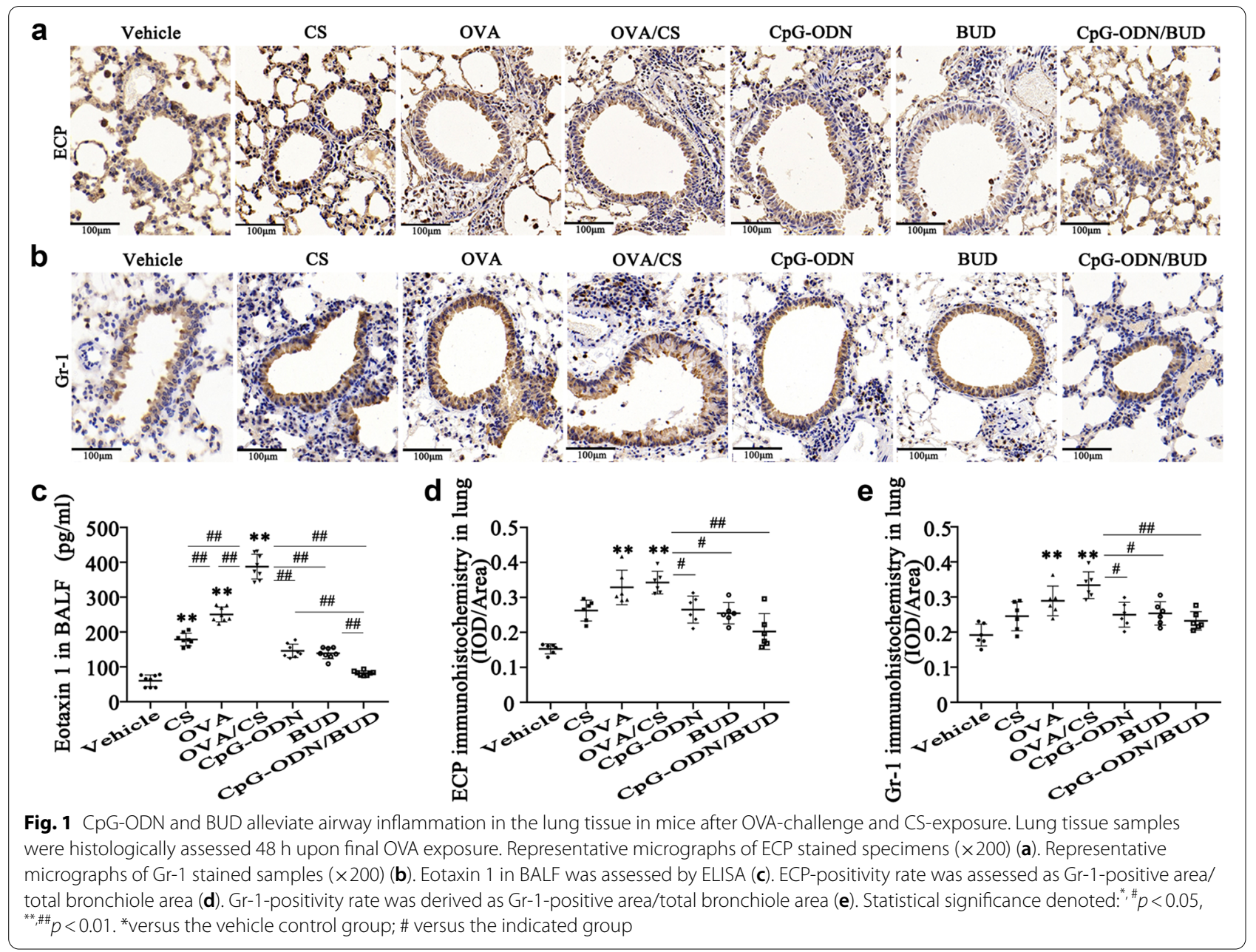

and CpG-ODNs and BUD synergistically decreased the exacerbated levels of Th17- and Th2-associated cytokine, and enhanced the biosynthesis of the Th1-associated cytokine IFN- $\gamma$.

\section{HDAC2 activity and expression restoration upon treatment with CpG-ODNs and BUD in CS-exposure asthmatic mice}

CS reduces responsiveness to steroids by modifying histone acetyltransferase, which is an essential epigenetic enzyme that mediates the anti-inflammatory effects of steroids [31, 32]. Furthermore, HDAC2 activity and levels are substantially decreased by oxidative/nitrative stress, causing insensitivity to the anti-inflammatory effects of GCs [33]. In this study, we assessed the levels of secreted HDAC2 in lung tissue samples by immunohistochemistry, ELISA and Western blotting. As shown in Fig. 3, OVA challenge and CS exposure both markedly decreased HDAC2 mRNA and protein levels (Fig. 3ae). We also investigated the effects of CpG-ODNs and BUD on CS-induced changes in HDAC2 mRNA and protein expression levels to verify whether CpG-ODNs affect HDAC2 expression. Interestingly, it was found that after treatment with CpG-ODNs or BUD only, the effect on HDAC2 gene expression levels were with the opposite of those in the untreated group, and this effect was enhanced after coadministration of CpG-ODN and BUD (Fig. 3a-c, e), however, a nonsignificant increase in HDAC2 protein expression levels was observed in mice that were administered CpG-ODN plus BUD $(p=0.06$, Fig. 3d).

Moreover, based on studies reporting that patients with severe asthma have diminished GCs sensitivity in peripheral blood monocytes (PBMCs) in comparison with patients with nonsevere asthma, in association with decreased HDAC2 activity that parallels the impairment in GCs sensitivity [34], we analyzed HDAC2 activity with an HDAC2 activity assay kit. As expected, similar to HDAC2 expression, HDAC2 activity in OVA + CS challenged mice was obviously suppressed and markedly recovered after the administration of CpG-ODN or BUD, with significant differences between the OVA/CS 


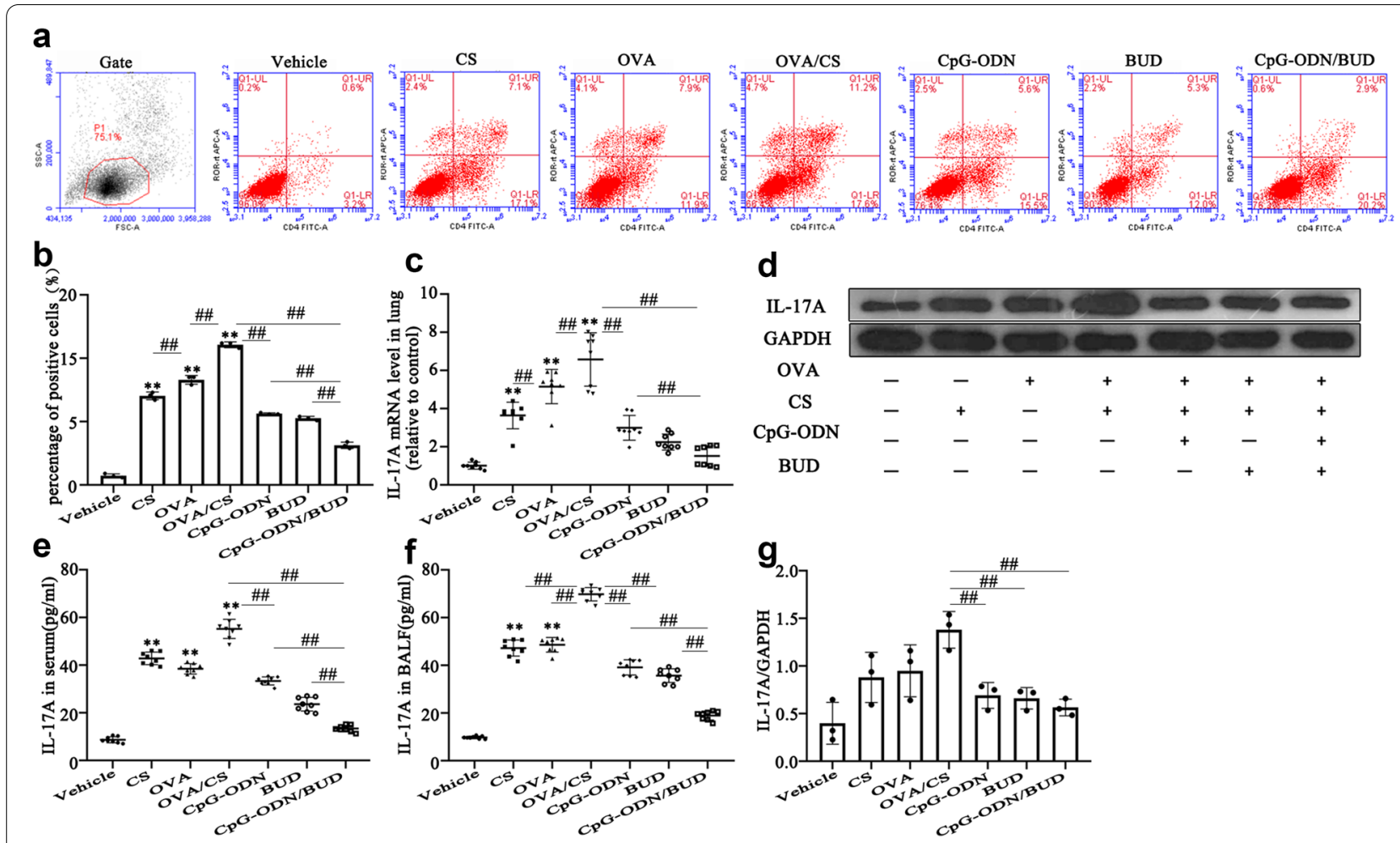

Fig. 2 CpG-ODN and BUD synergistically alter Th17 responses in the lower airway. RORyt ${ }^{+} C D 4^{+}$cells representing Th17 cells were assessed flow-cytometrically (a) and positive cells were quantitated (b). Th17-related cytokine amounts in serum (e) and BALF (f) were determinedby ELISA, and relative mRNA expression was measured by qRT-PCR in lung tissues from mice (c). Immunoblot was carried out for assessing the protein amounts of Th17-associated cytokinesinlung tissues, with GAPDH as a loading control (d); HDAC2/GAPDH ratios were assessed (g). Statistical significance denoted: ${ }^{*}, \#<0.05,{ }^{* *}, \# \#<0.01$. *versus the vehicle control group; \# versus the indicated group

and CpG-ODN/BUD groups, reflecting the changes in HDAC2 protein expression (Fig. 3f) .

These data suggested that the expression and activity of HDAC2 was impaired in chronic asthmatic murine models. Meanwhile, CpG-ODNs restored responsiveness to GCs therapy by restoring HDAC2 expression and enhancing HDAC2 activity. When combined with BUD, CpG-ODNs restored HDAC2 activity and expression more substantially than either CpG-ODNs or BUD alone.

\section{Decreased RORyt expression and Th17 responses in response to CS and OVA challenge after CpG-ODNs and BUD treatment}

HDAC2 is important in Th-17 cell differentiation from naive $\mathrm{CD}^{+} \mathrm{T}$ cells, and RORyt involvement in this process has attracted increasing attention [35, 36]. The catalytic activity of $\mathrm{HDAC} 2$ is important in inhibiting RORyt transcriptional activity, and SUMOylated ROR $\gamma \mathrm{t}$ recruits HDAC2 to the IL-17 promoter for gene downregulation [37]. To explore the mechanism by which CpG-ODNs treatment regulates the cytokine IL-17 A due to HDAC2 upregulation, we next examined the levels of ROR $\gamma \mathrm{t}$, which is an important biomarker of the
HDAC2-mediated Th17 response in CS-induced asthma, by immunohistochemistry, ELISA and Western blotting. The results showed a distinct increasing trend in ROR $\gamma t$ mRNA and protein expression levels in CS-exposed asthmatic mice in comparison with vehicle control mice (Fig. 4). Meanwhile, upon combined administration of CpG-ODNs and BUD, the animals showed significantly decreased RORyt mRNA and protein levels (Fig. 4), indicating that CpG-ODNs combined with BUD suppressed RORyt to a certain extent, thereby inhibiting IL-17 A expression in Th17 cells.

\section{CpG-ODNs and BUD synergistically regulate the interplay between HDAC2, RORyt and IL-17 A, orchestrating inflammatory reactions in $\mathrm{HBE}$ cells}

Airway epithelial cells play a critical role on in defense against allergens, viruses, and environmental pollutants, which are involved in asthma pathogenesis. Moreover, IL-17 A is found in airway epithelial cells [38]. To further confirm whether CpG-ODNs inhibit the RORytmediated Th17 response via HDAC2, we next examined in vitro cultures of $\mathrm{HBE}$ cells exposed to OVA and/or CSE, that were administered CpG-ODNs and/or BUD. 


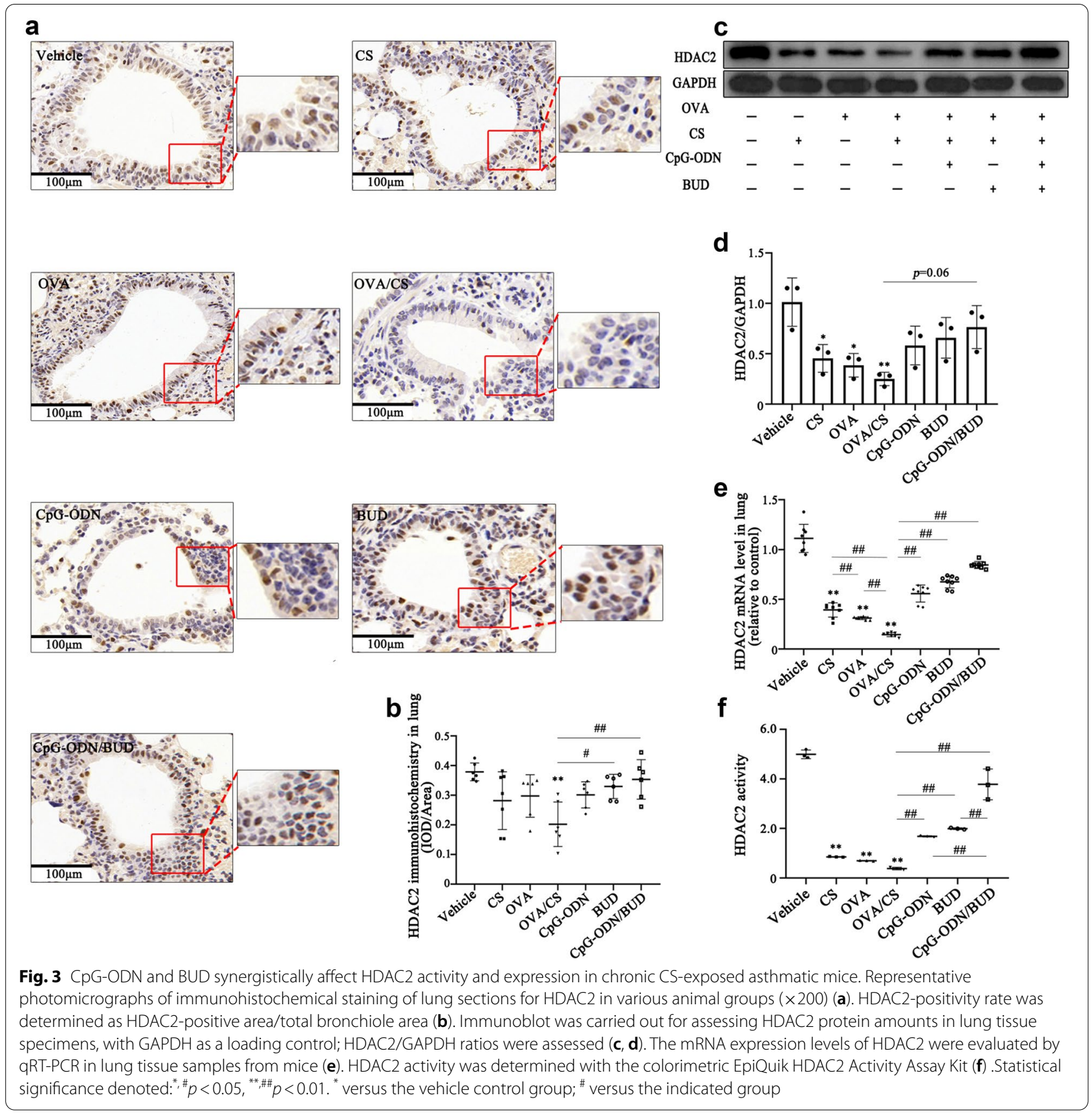

We performed ELISA, qRT-PCR, Western blotting, immunofluorescence analysis and flow cytometry to assess the levels of cytokines, HDAC2 and RORyt in all groups.

Consistent with the animal data, CSE-exposed or OVA-challenged HBE cells had elevated IL-5, IL-13 (Th2 cytokines) and IL-17 A (Th17 cytokine) levels compared with those in the vehicle control group. These cytokines were markedly increased in $\mathrm{HBE}$ cells after co-exposure to CSE and OVA (all $p<0.01$,
Fig. 5a-f). Moreover, HBE cells to OVA and CSE alone or in combination significantly reduced HDAC2 levels and markedly increased RORyt and IL-17 A gene and protein levels, suggesting specific associations of HDAC2 and ROR $\gamma$ t with the IL-17 promoter in HBE cells (all $p<0.05$, Figs. $5 g-h$ and 6). Since HDAC2 is the main HDAC that contributes to the effects of GCs, whether CpG-ODNs influence the interplay between HDAC2, RORyt and IL-17 A in HBE cells was examined. OVA- and CSE-exposed HBE cells were treated 


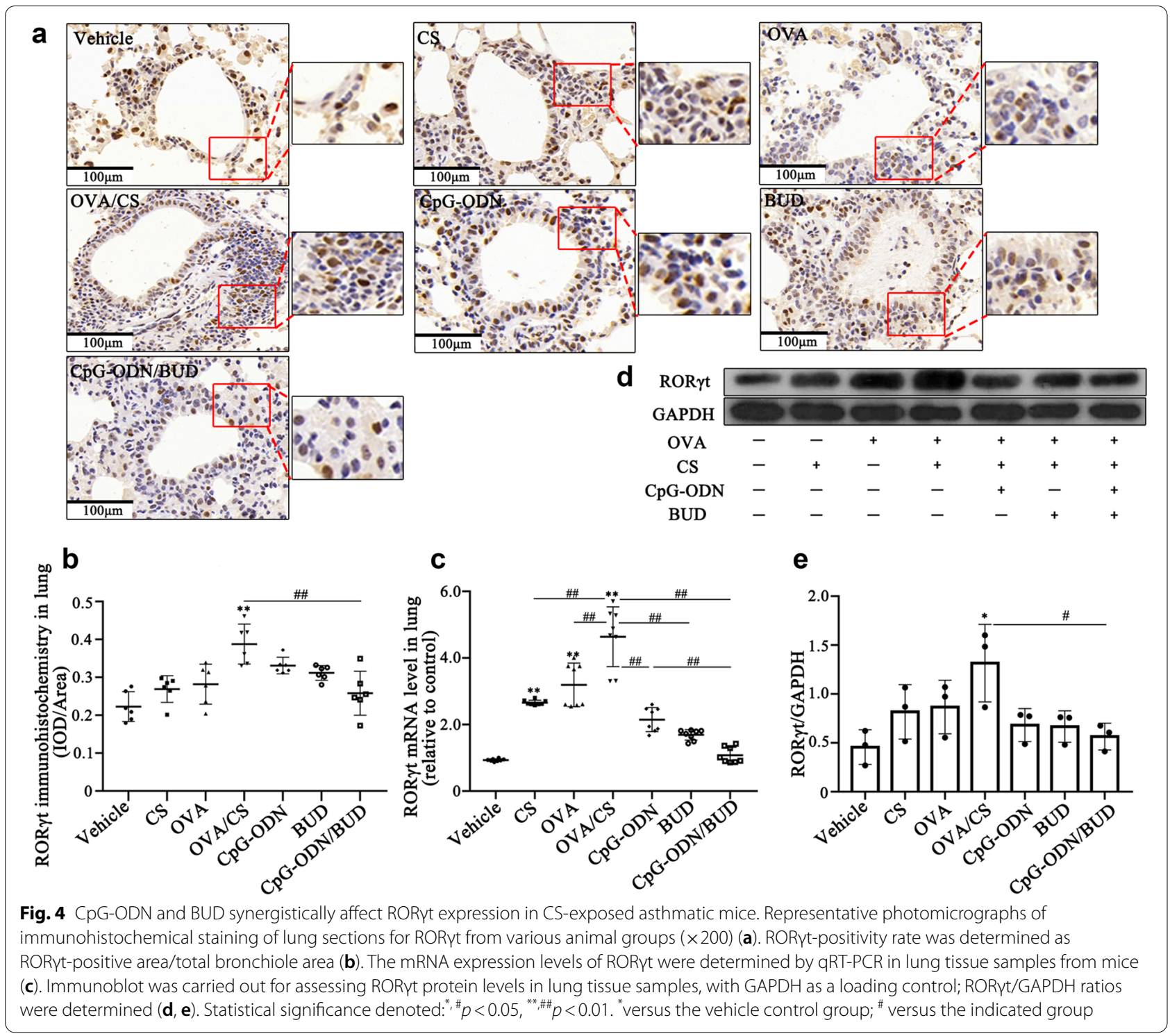

with CpG-ODNs and BUD. Interestingly, after administration of CpG-ODNs or BUD, HDAC2 protein levels showed an increasing trend. Notably, the increasing trend in HDAC2 expression changes was more pronounced after combined treatment with CpG-ODNs and BUD. However, contrary to the HDAC2 results, ROR $\gamma \mathrm{t}$ and IL-17 A levels were decreased in mice that were administered CpG-ODNs and were notably reduced after the co-administration of BUD and CpGODNs (all $p<0.05$, Figs. $5 \mathrm{~g}-\mathrm{h}$ and 6 ).

Collectively, these data provide convincing evidence of an interplay between HDAC2 and ROR $\gamma$ t in OVAinduced and CSE-exposed airway epithelial cells, which substantially affects allergic airway inflammation. Moreover, CpG-ODNs could partly affect this interplay, by simultaneously improving HDAC2 expression and inhibiting RORyt expression.

\section{Discussion}

Our previous study and others revealed that CS-exposed asthma exhibits elevated inflammatory cell infiltration $[10,31]$, mucus production, airway remodeling, and Th2/Th17 polarization, which was further confirmed in the current study (Figs. 1, 2 and Additional file 1: Figs. S3 and S4). As shown in Fig. 1, immunohistochemical analysis of Gr-1 (neutrophil-specific marker) and ECP (eosinophil-specific marker) confirmed a substantially increase in neutrophil and eosinophil influx into the lung, which suggested that CS and OVA induced the infiltration of inflammatory cells, including eosinophils 


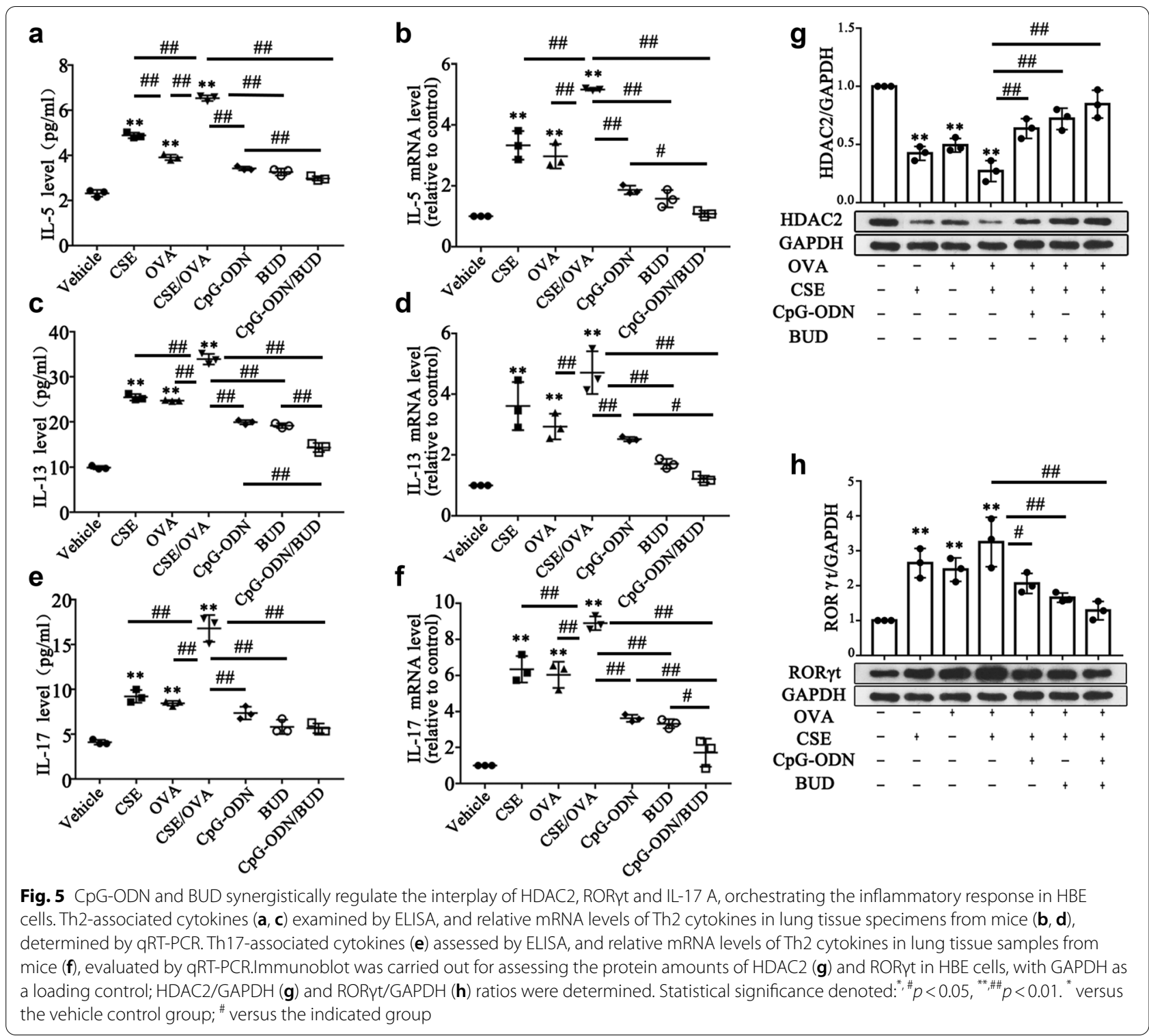

and neutrophils, into pulmonary tissues. Neutrophilic inflammation is driven by IL-17, TNF- $\alpha$, and IL-8 [39]. This study showed that IL-17 and neutrophil-associated chemokines, including IL- 8 and TNF- $\alpha$, were significantly elevated in the OVA/CS group, which may account for the enhanced infiltration of neutrophils into pulmonary tissue. In addition, eotaxin 1 was increased in the BALF in the CS/OVA group (Fig. 1). Eotaxin 1 promotes the recruitment of eosinophils and other immune cells, such as neutrophils [40]. Increasing evidence suggests that decreased sensitivity to GCs is associated with neutrophilic airway inflammation, and steroid-insensitive asthma is characterized by Th17 cytokines with neutrophilic inflammation [41, 42]. In this study, BUD alone markedly decreased Th2/Th17 cytokines in BALF and reduced immune cells in pulmonary tissue (Figs. 1, 2 and Additional file 1: Figs. S3 and S4), although to a lesser extent than that observed in our previous study [10]. These findings indicate that the current model is not GCs resistant but rather GCs insensitive in the context of lung cell inflammation. According to these findings, mice coexposed to CS and OVA showed exaggerated reactions to allergen inhalation, triggering inflammation that simultaneously involved eosinophils and neutrophils, elevated type 17-associated immune responses, and relative insensitivity to GCs.

Our previous study demonstrated that CpG-ODNs alleviate mixed airway neutrophil and eosinophil 

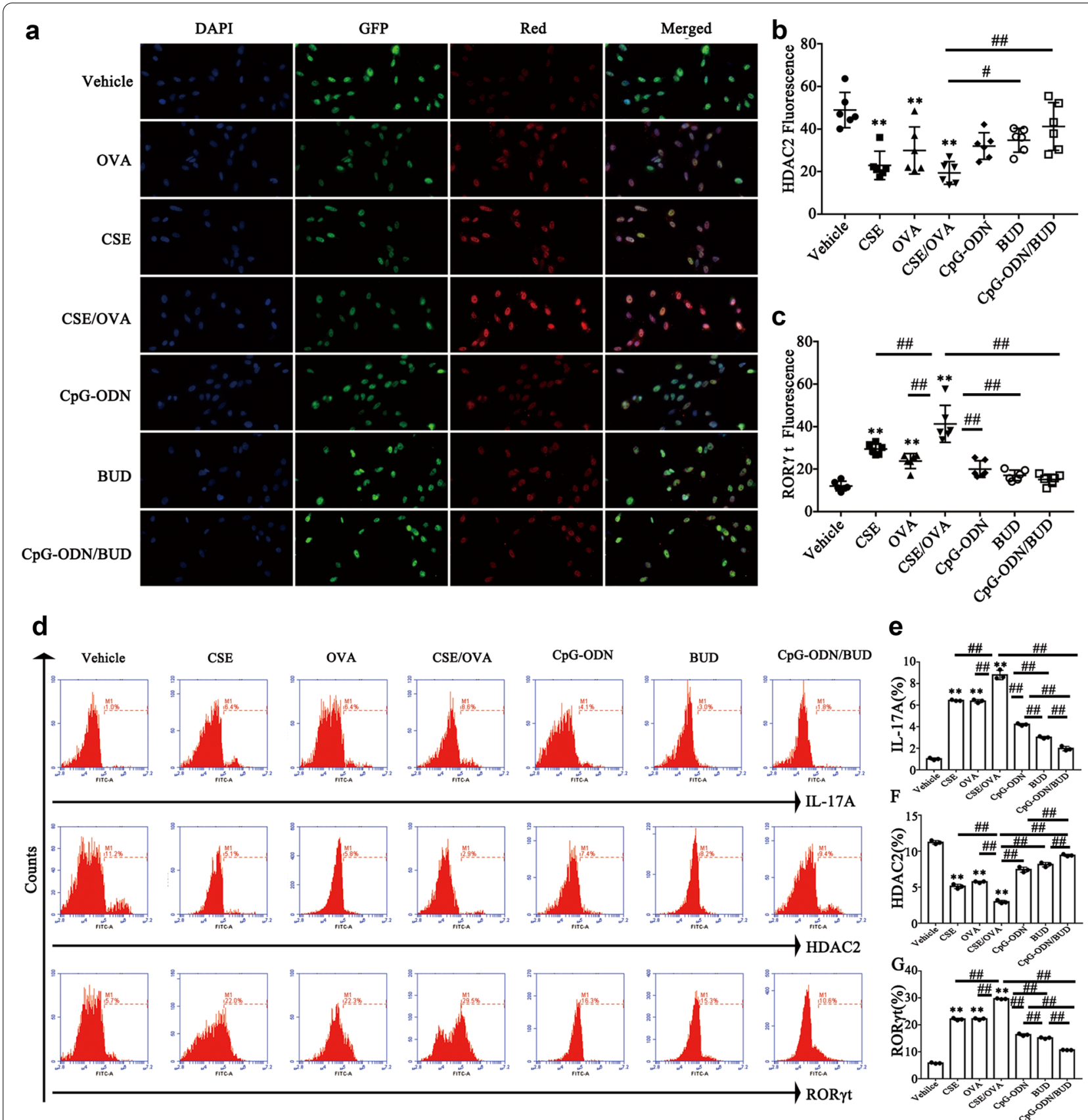

Fig. 6 CpG-ODN and BUD synergistically affect HDAC2, RORyt and IL-17 A in HBE cells. Representative micrographs of immunofluorescent staining of HBE cells for detecting tubulin GFP(green; HDAC2-positive) and H2BmCherry(Red; RORyt-positive) cells (×200) (a). HDAC2-positivity rate was determined as tubulin GFP(green)-positive area/total area (b). RORyt-positivity rate was evaluated as H2BmCherry(Red)-positive area/total area (c). Representative images depicting $\mathrm{IL}-17 \mathrm{~A}^{+}, \mathrm{HDAC2}^{+}$, and RORyt ${ }^{+}$cells in $\mathrm{HBE}$ cell populations (d). The ratios of specific protein-positive HBE cells to total HBE cells (e-g) were assessed by flow cytometry. Statistical significance denoted: ${ }^{*}, \#<0.05,{ }^{* *}, \# p<0.01$. ${ }^{*}$ versus the vehicle control group; ${ }^{\prime}$ versus the indicated group

inflammation in CS exposure OVA-induced asthma [10]. Several reports have revealed decreased HDAC2 activity in smokers and the sputum cells of patients with respiratory diseases, as well as in CS-exposed asthma mice
$[19,25,32,43]$, indicating that insufficient transcriptional corepressor levels and activity could be critical for asthma pathogenesis [44]. Emerging evidence has suggested that theophylline could downregulate the inflammatory 
response, locally and systemically, by increasing HDAC2 activity in patients with asthma $[15,45]$. Since CpG-ODNs reduce the inflammatory response, we examined whether CpG-ODNs modulate HDAC2 activity and expression, thereby enhancing the response to GCs in CS-exposure asthma mice. In the current CS exposure OVA-induced model, both HDAC2 mRNA and protein levels or activity were markedly suppressed, and as expected, these factors were increased after CpG-ODNs administration. Furthermore, the combination of CpG-ODNs and BUD more substantially restored HDAC2 activity and expression, which may account for the amelioration of corticosteroid insensitivity. Interestingly, consistent with another study [46], BUD robustly suppressed mediator release compared to the enhancement in HDAC2 activity [15, 47]. CpGODNs may act in an indirect manner with corticosteroids to increase its effect on HDAC2 activity, suggesting that CpG-ODNs exert corticosteroid-sparing effects.

There is increasing evidence that Th17 lymphocytes play a critical role in inducing neutrophilic airway inflammation. Th17 cells produce various inflammatory cytokines, including IL-17 A, which regulates cellular immunity by upregulating downstream proinflammatory molecules in epithelial and mesenchymal cells, thereby mediating neutrophil infiltration and activation, and promoting neutrophil accumulation in pulmonary tissues. Interestingly, in the present study, opposite trends in between HDAC2 and IL-17 A were found, with HDAC2 downregulated while IL-17 A was upregulated in CS-exposure asthmatic mice. Several studies have shown that the striking interaction between HDAC2 and IL-17 A forms a vicious cycle, leading to the exacerbation of asthma [43]. According to a study by Lai et al. [43], HDAC2 impairment upregulates IL-17 A, and IL-17 A deficiency ameliorates the reduction in HDAC2, suggesting that HDAC2 is a mediator that affects the secretion of IL-17 A, thereby causing a Th17polarized response. In the current study, CS-exposed mice that were administered CpG-ODNs had elevated HDAC2 expression and attenuated IL-17 A production. Collectively, CpG-ODNs is likely involved in the interaction between HDAC2 and IL-17 A. Therefore, the current results indicate that CpG-ODNs may affect IL-17 A secretion by modulating HDAC2 activity and expression, thereby inhibiting the Th17 response.

On the other hand, RORyt is an important transcription factor that regulates IL-17 A [48]. Singh et al. described a detailed mechanism by which the SUMOylation of RORyt promotes HDAC2 interactions with the IL-17 promoter and suppresses IL-17 A transcription [36]. Furthermore, a study demonstrated RORyt acetylation in Th17 cells and this effect was significantly enhanced by HDAC2 inhibitors [49], which was consistent with a recent study that revealed elevated RORyt acetylation in cultured CSE-induced HBE cells with HDAC2 silencing [25]. Based on these findings, HDAC2 may inhibit RORyt-mediated IL-17 A production, thereby attenuating the Th17 response. Consistent with our hypothesis, the results indicated a negative correlation between HDAC2 and RORyt expression in CSexposure asthmatic mice. Therefore, we hypothesized that CpG-ODNs might mainly accelerate the binding capacity of RORyt to IL-17 A via HDAC2, to inhibit IL-17 A expression.

Bronchial epithelial cells are increasingly thought to contribute to innate immunity. We hypothesized that altered sensitivity to GCs in airway epithelial cells is substantially involved in GCs insensitivity in inflammatory responses [50]. Consistent with a recent study [9], we found that CSE exposure markedly downregulated HDAC2 expression in HBE cells, and costimulation with OVA and CSE in asthmatic conditions further deceased HDAC2 expression (Figs. 5 and 6). Contrary to the change in HDAC2 expression, elevated RORyt expression was observed in HBE cells cultured with CSE, with markedly increased expression in HBE cells that were administered both CSE and OVA (Figs. 5 and 6). These results indicated an interplay between HDAC2 and RORyt in OVA- and CSE-induced airway epithelial cells, suggesting an essential function of these factors in Th17 inflammation. Next, whether CpG-ODNs regulate HDAC2 and RORyt in airway epithelial cells to change the sensitivity of these cells to GCs was investigated. We treated HBE cells induced with OVA and CSE with CpG-ODNs and BUD and found that CpG-ODNs regulated the interplay between HDAC2 and RORyt, synergistically with BUD to some extent, which was consistent with the effects in the CS-exposure asthmatic murine model (Figs. 5 and 6). Of note, due to relatively expensive, low storage temperature, and possible biosafety consideration, we used complete culture medium supplemented with $10 \%$ FBS, instead of the growth medium recommended by the ATCC, to maintain HBE cells in vitro, which may be a potential limitation of this study. However, HBE cells showed uniform and stable growth in a continuous and consistent manner (Additional file 1: Fig. S2), demonstrating that HBE cells were cultured successfully. It is known that IL-17 A modulates the protective effects of HDAC2 on airway inflammation in asthma and that HDAC2 activation and/or IL-17 A downregulation can prevent allergic airway inflammation [43]. Moreover, RORyt transcriptionally upregulates IL-17 A, indicating that CpG-ODNs may suppress RORyt-mediated IL-17 A expression via HDAC2 in vitro, which is consistent with the above evidence.

\section{Conclusions}

Overall, CpG-ODNs and BUD synergistically improved adverse CS-exposure asthma outcomes and inhibited, at least in part, RORyt-mediated Th17 response by restoring 
HDAC2 expression and activity, consequently ameliorating GCs insensitivity. These data suggest that CpG-ODNs may have therapeutic value in reviving steroid effects in CSexposure asthma, providing new insights into the mechanism by which CpG-ODNs improve sensitivity to steroids.

\section{Supplementary Information}

The online version contains supplementary material available at https://doi. org/10.1186/s13578-021-00607-3.

Additional file 1: Table S1. Sequence of primers for qRT-PCR. Fig. S1. Experimental protocol for the study. Fig. S2. HBE Cell viability varies with increasing concentration and time. Fig. S3. CpG-ODNs and BUD inhibit mucus secretion and airway structural remodeling in mice induced by OVA-challenge and CS-exposure. Fig. S4. CpG-ODNs and BUD synergistically alter Th1/Th2 type responses in the lower airway.

\section{Acknowledgements}

The authors express their gratitude to Bing Xie for help in establishing the mouse model. We would like to thank Ju Jiao for administrative assistance. The current manuscript was revised by a native English speaking editor at American Journal Experts.

\section{Authors' contributions}

Conceived and designed the experiments: LHT, YQM, ZTT. Performed the experiments: LHT, YQM, LYS, YXN, ZXL, YHL, WWB, MP. Analyzed the data: $L H T$, YQM, LYS, YXN, ZTT. Wrote the paper: LHT, YQM, LYS, YXN. All authors read and approved the final manuscript.

\section{Funding}

The present work was funded by the National Natural Science Foundation of China (No. 81973984, 81470220 and 81970017), Guangdong Basic and Applied Basic Research Foundation (No.2019A1515010918), the Science and Technology Program of Guangzhou, China (No.201707010076), and the Science and Technology Planning Project of Guangdong Province, China (No.2016A020215220).

\section{Availability of data and materials}

The datasets used and/or analyzed during the current study are available from the corresponding author on reasonable request.

\section{Declarations}

\section{Ethics approval and consent to participate}

All animal assays were approved by the ethics committee of the Third Affiliated Hospital of Sun-Yat-Sen University.

\section{Consent for publication}

All the co-authors consent to publish the work in Cell \& Bioscience.

\section{Competing interests}

The authors declare that they have no competing interests.

Received: 25 July 2020 Accepted: 7 May 2021

Published online: 20 May 2021

\section{References}

1. Mei $D$, Tan WSD, Wong WSF. Pharmacological strategies to regain steroid sensitivity in severe asthma and COPD. Curr Opin Pharmacol. 2019:46:73-81.

2. Thomson NC, Shepherd M, Spears M, Chaudhuri R. Corticosteroid insensitivity in smokers with asthma: clinical evidence, mechanisms, and management. Treat Respir Med. 2006;5:467-81.
3. Belvisi MG, Baker K, Malloy N, Raemdonck K, Dekkak B, Pieper M, Nials AT, Birrell MA. Modelling the asthma phenotype: impact of cigarette smoke exposure. Respir Res. 2018;19:89.

4. Tomlinson JE, MCMahon AD, Chaudhuri R, Thompson JM, Wood SF, Thomson NC. Efficacy of low andhigh dose inhaled corticosteroid in smokers versus non-smokers with mild asthma. Thorax. 2005;60:282-7.

5. Chaudhuri R, Livingston E, McMahon AD, Thomson L, Borland W, Thomson NC. Cigarette smoking impairs the therapeutic response to oral corticosteroids in chronic asthma. Am J Respir Crit Care Med. 2003;168:1308-11.

6. Mukherjee M, Svenningsen S, Nair P. Glucocortiosteroidsubsensitivity and asthma severity. Curr Opin Pulm Med. 2017;23:78-88.

7. Hansbro PM, Kaiko GE, Foster PS. Cytokine/anti-cytokine therapy - novel treatments for asthma? Br J Pharmacol. 2011:163:81-95.

8. Ordoñez CL, Shaughnessy TE, Matthay MA, Fahy JV. Increased neutrophil numbers and IL-8 levels in airway secretions in acute severe asthma: Clinical and biologic significance. Am J Respir Crit Care Med. 2000;161:1185-90.

9. Luo Q, Lin J, Zhang L, Li H, Pan L. The anti-malaria drug artesunate inhibits cigarette smoke and ovalbumin concurrent exposure-induced airway inflammation and might reverse glucocorticoid insensitivity. Int Immunopharmacol. 2015;29:235-45.

10. Li HT, Lin YS, Ye QM, Yang XN, Zou XL, Yang HL, Zhang TT. Airway inflammation and remodeling of cigarette smoking exposure ovalbumininduced asthma is alleviated by $\mathrm{CpG}$ oligodeoxynucleotides via affecting dendritic cell-mediated Th17 polarization. Int Immunopharmacol. 2020;82:106361.

11. Zhang $H$, Liu Q, Kong L, Xu S. Mucin 1 downregulation impairs the antinecroptotic effects of glucocorticoids in human bronchial epithelial cells. Life Sci. 2019;221:168-77.

12. Ammit AJ. Glucocorticoid insensitivity as a source of drug targets for respiratory disease. Curr Opin Pharmacol. 2013;13:370-6.

13. Li M, Zhong X, He Z, Wen M, Li J, Peng X, Liu G, Deng J, Zhang J, Bai J. Effect of erythromycin on cigarette-induced histone deacetylase protein expression and nuclear factor-kB activity in human macrophages in vitro. Int Immunopharmacol. 2012;12:643-50.

14. Ito K, Yamamura S, Essilfie-Quaye S, Cosio B, Ito M, Barnes PJ, Adcock IM Histone deacetylase 2-mediated deacetylation of the glucocorticoid receptor enables NF-kappaB suppression. J Exp Med. 2006;203:7-13.

15. Cosío BG, Mann B, Ito K, Jazrawi E, Barnes PJ, Chung KF, Adcock IM. Histone acetylase and deacetylase activity in alveolar macrophages and blood mononocytes in asthma. Am J Respir Crit Care Med. 2004;170:141-7.

16. Kobayashi Y, Bossley C, Gupta A, Akashi K, Tsartsali L, Mercado N, Barnes PJ, Bush A, Ito K. Passive smoking impairs histone deacetylase-2 in children with severe asthma. Chest. 2014;145:305-12.

17. Lai T, Chen M, Deng Z, LY, Wu D, Li D, Wu B. YKL-40 is correlated with FEV1 and the asthma control test (ACT) in asthmatic patients: influence of treatment. BMC Pulm Med. 2015:15:1.

18. Malhotra D, Thimmulappa RK, Mercado N, Ito K, Kombairaju P, Kumar S, Ma J, Feller-Kopman D, Wise R, Barnes P, Biswal S. Denitrosylation of HDAC2 by targeting Nrf2 restores glucocorticosteroid sensitivity in macrophages from COPD patients. J Clin Invest. 2011;121:4289-302.

19. Ito K, Lim S, Caramori G, Chung KF, Barnes PJ, Adcock IM. Cigarette smoking reduces histone deacetylase 2 expression, enhances cytokine expression, and inhibits glucocorticoid actions in alveolar macrophages. Faseb J. 2001:15:1110-2.

20. To Y, Ito K, Kizawa Y, Failla M, Ito M, Kusama T, Elliott WM, Hogg JC, Adcock IM, Barnes PJ. Targeting phosphoinositide-3-kinase-delta with theophylline reverses corticosteroid insensitivity in chronic obstructive pulmonary disease. Am J Respir Crit Care Med. 2010;182:897-904.

21. Marwick JA, Caramori G, Stevenson CS, Casolari P, Jazrawi E, Barnes PJ, Ito K, Adcock IM, Kirkham PA, Papi A. Inhibition of PI3Kdelta restores glucocorticoid function in smoking-induced airway inflammation in mice. Am J Respir Crit Care Med. 2009;179:542-8.

22. Casale TB, Cole J, Beck E, Vogelmeier CF, Willers J, Lassen C, HammannHaenni A, Trokan L, Saudan P, Wechsler ME. CYT003, a TLR9 agonist, in persistent allergic asthma - a randomized placebo-controlled Phase $2 b$ study. Allergy. 2015;70:1160-8.

23. Senti G, Johansen P, Haug S, Bull C, Gottschaller C, Müller P, Pfister T, Maurer P, Bachmann MF, Graf N, Kündig TM. Use of A-type CpG 
oligodeoxynucleotides as an adjuvant in allergen-specific immunotherapy in humans: a phase I/lla clinical trial. Clin Exp Allergy. 2009;39:562-70.

24. Hanagata N. CpG oligodeoxynucleotide nanomedicines for the prophylaxis or treatment of cancers, infectious diseases, and allergies. Int J Nanomedicine. 2017;12:515-31.

25. Lai T, Tian B, Cao C, Hu Y, Zhou J, Wang Y, Wu Y, Li Z, Xu X, Zhang M, Xu F, Cao Y, Chen M, Wu D, Wu B, Dong C, Li W, Ying S, Chen Z, Shen H. HDAC2 Suppresses IL17A-Mediated Airway Remodeling in Human and Experimental Modeling of COPD. Chest. 2018;153:863-75.

26. Song Y, Chi DY, Yu P, Lu JJ, Xu JR, Tan PP, Wang B, Cui YY, Chen HZ. Carbocisteine improves histone deacetylase 2 deacetylation activity via regulating sumoylation of histone Deacetylase 2 in human tracheobronchial epithelial cells. Front Pharmacol. 2019;10:166.

27. Zhou M, Jiao L, Liu Y. sFRP2 promotes airway inflammation and Th17/ Treg imbalance in COPD via Wnt/beta-catenin pathway. Respir Physiol Neurobiol. 2019;270:103282

28. Alcorn JF, Crowe CR, Kolls JK. TH17 cells in asthma and COPD. Annu Rev Physiol. 2010;72:495-516.

29. McKinley L, Alcorn JF, Peterson A, Dupont RB, Kapadia S, Logar A, Henry A, Irvin CG, Piganelli JD, Ray A, Kolls JK. TH17 cells mediate steroid-resistant airway inflammation and airway hyperresponsiveness in mice. J Immunol. 2008;181:4089-97.

30. Liao W, Tan WS, Wong WS. Andrographolide restores steroid sensitivity to block lipopolysaccharide/IFN-gamma-Induced IL-27 and airway hyperresponsiveness in mice. J Immunol. 2016;196:4706-12.

31. Xia M, Xu H, Dai W, Zhu C, Wu L, Yan S, Ge X, Zhou W, Chen C, Dai Y. The role of HDAC2 in cigarette smoke-induced airway inflammation in a murine model of asthma and the effect of intervention with roxithromycin. J Asthma. 2018;55:337-44.

32. Barnes PJ. Glucocorticosteroids. Handb Exp Pharmacol. 2017;237:93-115.

33. Hew M, Bhavsar P, Torrego A, Meah S, Khorasani N, Barnes PJ, Adcock I, Chung KF. Relative corticosteroid insensitivity of peripheral blood mononuclear cells in severe asthma. Am J Respir Crit Care Med. 2006;174:134-41.

34. Ivanov II, McKenzie BS, Zhou L, Tadokoro CE, Lepelley A, Lafaille JJ, Cua DJ, Littman DR. The orphan nuclear receptor RORgammat directs the differentiation program of proinflammatory $\mathrm{IL}-17+\mathrm{T}$ helper cells. Cell. 2006;126:1121-33.

35. Manel N, Unutmaz D, Littman DR. The differentiation of human $T(H)-17$ cells requires transforming growth factor-beta and induction of the nuclear receptor RORgammat. Nat Immunol. 2008;9:641-9.

36. Singh AK, Khare P, Obaid A, Conlon KP, Basrur V, DePinho RA, Venuprasad K. SUMOylation of ROR-gammat inhibits IL-17 expression and inflammation via HDAC2. Nat Commun. 2018:9:4515.

37. Montalbano AM, Riccobono L, Siena L, Chiappara G, Di Sano C, Anzalone G, Gagliardo R, Ricciardolo FLM, Sorbello V, Pipitone L, Vitulo P, Profita M. Cigarette smoke affects IL-17A, IL-17F and IL-17 receptor expression in the lung tissue: Ex vivo and in vitro studies. Cytokine. 2015;76:391-402.

38. Thomson NC, Chaudhuri R, Livingston E. Asthma and cigarette smoking. Eur Respir J. 2004;24:822-33.
39. Barnes PJ. Severe asthma: advances in current management and future therapy. J Allergy Clin Immunol. 2012;129:48-59.

40. Srivastava KD, Dunkin D, Liu C, Yang N, Miller RL, Sampson HA, Li XM. Effect of Antiasthma Simplified Herbal Medicine Intervention on neutrophil predominant airway inflammation in a ragweed sensitized murine asthma model. Ann Allergy Asthma Immunol. 2014;112:339-47. e331-332.

41. Chang HS, Lee TH, Jun JA, Baek AR, Park JS, Koo SM, Kim YK, Lee HS, Park CS. Neutrophilic inflammation in asthma: mechanisms and therapeutic considerations. Expert Rev Respir Med. 2017;11:29-40.

42. Essilfie AT, Horvat JC, Kim RY, Mayall JR, Pinkerton JW, Beckett EL, Starkey MR, Simpson JL, Foster PS, Gibson PG, Hansbro PM. Macrolide therapy suppresses key features of experimental steroid-sensitive and steroidinsensitive asthma. Thorax. 2015;70:458-67.

43. Lai T, Wu M, Zhang C, Che L, Xu F, Wang Y, Wu Y, Xuan N, Cao C, Du X, Wu $B$, Li W, Ying S, Shen H, Chen Z. HDAC2 attenuates airway inflammation by suppressing IL-17A production in HDM-challenged mice. Am J Physiol Lung Cell Mol Physiol. 2019;316:L269-79.

44. Wadhwa R, Dua K, Adcock IM, Horvat JC, Kim RY, Hansbro PM. Cellular mechanisms underlying steroid-resistant asthma. Eur Respir Rev. 2019;28:190096.

45. Yao H, Rahman I. Role of histone deacetylase 2 in epigenetics and cellular senescence: implications in lung inflammaging and COPD. Am J Physiol Lung Cell Mol Physiol. 2012;303:L557-66.

46. An TJ, Rhee CK, Kim JH, Lee YR, Chon JY, Park CK, Yoon HK. Effects of macrolide and corticosteroid in neutrophilic asthma mouse model. Tuberc Respir Dis (Seoul). 2018:81:80-7.

47. Perng DW, Su KC, Chou KT, Wu YC, Chen CS, Hsiao YH, Tseng CM, Chen YH, Hsueh TY, Lee YC. Long-acting beta2 agonists and corticosteroids restore the reduction of histone deacetylase activity and inhibit $\mathrm{H}_{2} \mathrm{O} 2-$ induced mediator release from alveolar macrophages. Pulm Pharmacol Ther. 2012, 25:312-318.

48. Ichiyama K, Yoshida H, Wakabayashi Y, Chinen T, Saeki K, Nakaya M, Takaesu G, Hori S, Yoshimura A, Kobayashi T. Foxp3 inhibits RORgammatmediated IL-17A mRNA transcription through direct interaction with RORgammat. J Biol Chem. 2008;283:17003-8.

49. Wu Q, Nie J, Gao Y, Xu P, Sun Q, Yang J, Han L, Chen Z, Wang X, Lv L, Tsun A, Shen J, Li B. Reciprocal regulation of RORgammat acetylation and function by p300 and HDAC1. Sci Rep. 2015;5:16355.

50. Zijlstra GJ, Ten Hacken NH, Hoffmann RF, van Oosterhout AJ, Heijink IH. Interleukin-17A induces glucocorticoid insensitivity in human bronchial epithelial cells. Eur Respir J. 2012;39:439-45.

\section{Publisher's Note}

Springer Nature remains neutral with regard to jurisdictional claims in published maps and institutional affiliations.

Ready to submit your research? Choose BMC and benefit from:

- fast, convenient online submission

- thorough peer review by experienced researchers in your field

- rapid publication on acceptance

- support for research data, including large and complex data types

- gold Open Access which fosters wider collaboration and increased citations

- maximum visibility for your research: over $100 \mathrm{M}$ website views per year

At BMC, research is always in progress.

Learn more biomedcentral.com/submissions 